\title{
Eficiência fotoquímica de Nopalea cochenillifera Salm-Dyck em função da cobertura do solo em diferentes horários
}

Photochemical efficiency of Nopalea cochenillifera Salm-Dyck as a function of soil cover at different times

Eficiencia fotoquímica de Nopalea cochenillifera Salm-Dyck en función de la cobertura del suelo en diferentes momentos

\author{
José Thyago Aires Souza \\ ORCID: https://orcid.org/0000-0002-1508-1136 \\ Instituto Nacional do Semiárido, Brasil \\ E-mail: thyago.agro@hotmail.com \\ João Everthon da Silva Ribeiro \\ ORCID: https://orcid.org/0000-0002-1937-0066 \\ Universidade Estadual do Maranhão, Brasil \\ E-mail: j.everthon@hotmail.com \\ João Paulo de Farias Ramos \\ ORCID: https://orcid.org/0000-0001-6159-5026 \\ Universidade Federal da Paraíba, Brasil \\ E-mail: joaopaulodeframosjp@gmail.com \\ Jucilene Silva Araújo \\ ORCID: https://orcid.org/0000-0003-3811-2297 \\ Instituto Nacional do Semiárido, Brasil \\ E-mail: jucilene.araujo@insa.gov.br \\ Thiago Costa Ferreira \\ ORCID: https://orcid.org/0000-0002-2368-6223 \\ Instituto Nacional do Semiárido, Brasil \\ E-mail: thiago.ferreira@insa.gov.br \\ Raucha Carolina de Oliveira \\ ORCID: https://orcid.org/0000-0002-3517-8840 \\ Universidade Federal da Campina Grande, Brasil \\ E-mail: rauchaoliveira@gmail.com
}

\begin{abstract}
Resumo
A palma forrageira é a principal cultura xerófila cultivada no Brasil, entretanto seu crescimento e produção podem ser influenciados pelo desempenho fotoquímico do Fotossistema II, independentemente do sistema de cultivo. Neste contexto objetivou-se avaliar a eficiência fotoquímica de Nopalea cochenillifera Salm-Dyck em função da cobertura do solo em diferentes horários de avaliação. A pesquisa foi realizada na Estação Experimental Pendência, pertencente à Empresa Paraibana de Pesquisa, Extensão Rural e Regularização Fundiária (EMPAER-PB), município de Soledade, estado da Paraíba, Brasil. Os tratamentos foram distribuídos em blocos ao acaso, com três repetições no esquema fatorial $2 \times 4$, correspondente ao solo sem e com cobertura morta e quatro horários de avaliação da fluorescência da clorofila a, 08:00, 11:00, 14:00 e 17:00 horas. Foram analisadas as variáveis: fluorescência inicial, fluorescência variável, fluorescência máxima, relação e rendimento quântico potencial. O uso de cobertura morta elevou as fluorescências máxima e variável, especialmente nos primeiros horários de avaliação, a atividade fotoquímica do fotossistema II foi mais elevada às 17:00 horas.
\end{abstract}

Palavras-chave: Xerófila; Palma forrageira; Manejo de solo; Rendimento quântico.

\section{Abstract}

The forage spineless cacti are the main xerophilic crop grown in Brazil, however its growth and production may be influenced by the photochemical performance of Photosystem II, regardless of the cultivation system. In this context the objective was to evaluate the photochemical efficiency of Nopalea cochenillifera Salm-Dyck depending on ground cover at different schedules of evaluation. The research was carried out at the Pendência Experimental Station, belonging to the Paraibana Company for Research, Rural Extension and Regularization Landholding (EMPAER-PB), municipality of Soledade, state of Paraíba, Brazil. The treatments were distributed in randomized blocks, with three repetitions in the $2 \times 4$ factorial scheme, corresponding to the soil with and without mulch and four chlorophyll fluorescence evaluation times at, 08:00, 11:00, 14:00 and 17:00 hours. The variables analyzed were: initial 
fluorescence, variable fluorescence, maximum fluorescence, variable/initial fluorescence ratio and potential quantum yield. The use of mulch increased the maximum and variable fluorescence, especially in the first schedules of evaluation, photosystem II photochemical activity was highest at 17:00 hours.

Keywords: Xerophyte; Forage spineless cacti; Soil management; Quantum yield.

\section{Resumen}

El cactus forrajero es el principal cultivo xerófilo cultivado en Brasil, sin embargo, su crecimiento y producción pueden verse influenciados por el comportamiento fotoquímico del Fotosystem II, independientemente del sistema de cultivo. En este contexto, el objetivo fue evaluar la eficiencia fotoquímica de Nopalea cochenillifera Salm-Dyck en función de la cobertura del suelo en diferentes momentos de evaluación. La investigación se llevó a cabo en la Estación Experimental Pendência, perteneciente a la Empresa de Investigación, Extensión Rural y Regularización de Tierras de Paraíba (EMPAER-PB), município de Soledade, estado de Paraíba, Brasil. Los tratamientos se distribuyeron en bloques al azar, con tres repeticiones en un esquema factorial $2 \times 4$, correspondientes a suelo sin y con mulch y cuatro tiempos de evaluación de fluorescencia de clorofila a las 08:00, 11:00, 14:00 y 17:00. horas. Se analizaron las variables: fluorescencia inicial, fluorescencia variable, fluorescencia máxima, relación y rendimiento cuántico potencial. El uso de mantillo aumentó la fluorescencia máxima y variable, especialmente en las primeras horas de evaluación, la actividad fotoquímica del fotosistema II fue mayor a las 17:00 horas.

Palabras clave: Xerofilia; Cactus forrajeros; Manejo del suelo; Rendimiento cuántico.

\section{Introdução}

No Semiárido brasileiro, a produção de biomassa a partir de forrageiras xerófilas é a principal fonte de alimentação animal, especialmente de ruminantes, dentre as culturas xerófilas a palma forrageira destaca-se pelo seu alto potencial produtivo, além de possuir elevada rusticidade, característica indispensável para qualquer planta a ser cultivada nas zonas secas (Almeida et al., 2019).

Nestes ambientes, ainda são poucos os estudos fisiológicos sobre plantas regidas pelo Metabolismo Ácido das Crassuláceas (MAC), como a palma forrageira, especialmente no que diz respeito a rotas metabólicas da fluorescência da clorofila a. Este importante fator pondera a produção básica de fotoassimilados por meio do funcionamento do fotossistema II (PSII), sendo um indicador eficiente do uso dos recursos disponíveis pelas plantas e sua condição em relação a momentos de estresse (Ribeiro et al., 2019).

$\mathrm{Na}$ fase fotoquímica da fotossíntese, os elétrons ejetados dos pigmentos fotossintetizantes não utilizados na produção de ATP e NADPH através dos fotossistemas retornam aos pigmentos emitindo a luz absorvida, na forma de fluorescência e calor (Taiz et al., 2017). O sinal básico da fluorescência possui níveis característicos, que refletem o "status" da planta naquele momento, em relação ao seu próprio metabolismo e deste com o ambiente em que se encontra (Vieira et al., 2010). Assim sendo, a manutenção da integridade da rota metabólica da fluorescência da clorofila pode ser utilizada como um marcador biológico útil para diagnosticar efeitos de estresses em qualquer espécie vegetal, inclusive nesta cactácea (Souza et al., 2019).

Em meio às condições climáticas do Semiárido, as altas temperaturas e os longos períodos sem chuva que normalmente ultrapassam os 240 dias do ano podem propiciar potenciais estresses às plantas, inclusive sobre as taxas de reações químicas das células, causando danos nos aparatos fotossintéticos e de fotoinibição (Lopes, 2016). Para amenização dos efeitos da temperatura e redução da umidade edáfica o uso de técnicas conservacionistas como a cobertura morta é essencial, uma vez que contribui para o desenvolvimento das culturas, reduz a perda de água e a temperatura no perfil do solo, diminui a erosão superficial e incrementa a umidade, sendo uma prática muito recomendada para as regiões semiáridas (Borges et al., 2014).

Por consequência deste maior conforto, Sun et al. (2018) ressaltam que nestas regiões, o uso da cobertura edáfica com restos vegetais pode propiciar maior dinâmica fotoquímica e eficiência no uso da água pelas plantas. Neste contexto, objetivouse avaliar a eficiência fotoquímica de Nopalea cochenillifera Salm-Dyck em função da cobertura do solo em diferentes horários de avaliação. 


\section{Metodologia}

O experimento foi realizado em julho de 2018 sob condições de campo na Estação Experimental Pendência (EEPEN), pertencente à Empresa Paraibana de Pesquisa, Extensão Rural e Regularização Fundiária (EMPAER), no município de Soledade-PB. Esta localidade apresenta clima classificado como BSh, com o período chuvoso compreendido entre março e junho, temperaturas médias anuais próximo a $30{ }^{\circ} \mathrm{C}$, umidade relativa do ar em volta de $68 \%$ e precipitação média de 388,2 milímetros por ano (Alvares et al., 2013).

$\mathrm{Na}$ área experimental o solo foi classificado como Neossolo Flúvico (Embrapa, 2013), este foi caracterizado quanto às suas características físicas e químicas, para a profundidade de 0-20 cm, no Laboratório de Solos do Departamento de Engenharia Rural do Centro de Ciências Agrárias, Universidade Federal da Paraíba, Areia-PB (Tabela 1), de acordo com a metodologia proposta por Santos et al. (2017).

Tabela 1. Atributos químicos e físicos do solo na área experimental para a camada de 0-20 cm de um Neossolo Flúvico, Soledade-PB.

\begin{tabular}{|c|c|c|c|}
\hline \multicolumn{4}{|c|}{ CARACTERIZAÇÃO DO SOLO } \\
\hline ATRIBUTOS DA & VALOR & ATRIBUTOS FÍSICOS & VALOR \\
\hline FERTILIDADE & & & \\
\hline $\mathrm{pH}$ & 6,2 & DS $\left(\mathrm{kg} \mathrm{dm}^{-3}\right)$ & 1,31 \\
\hline $\mathrm{P}\left(\mathrm{mg} \mathrm{dm}^{-3}\right)$ & 70,3 & $\mathrm{DP}\left(\mathrm{kg} \mathrm{dm}^{-3}\right)$ & 2,68 \\
\hline $\mathrm{K}\left(\mathrm{cmol}_{\mathrm{c}} \mathrm{dm}^{-3}\right)$ & 0,21 & Areia $\left(\mathrm{g} \mathrm{kg}^{-1}\right)$ & 642 \\
\hline $\mathrm{Na}^{+}\left(\mathrm{cmol}_{\mathrm{c}} \mathrm{dm}^{-3}\right)$ & 0,16 & Silte $\left(\mathrm{g} \mathrm{kg}^{-1}\right)$ & 168 \\
\hline $\mathrm{H}^{+}+\mathrm{Al}^{3+}\left(\mathrm{cmol}_{\mathrm{c}} \mathrm{dm}^{-3}\right)$ & 2,51 & Argila $\left(\mathrm{g} \mathrm{kg}^{-1}\right)$ & 190 \\
\hline $\mathrm{Al}^{3+}\left(\mathrm{cmol}_{\mathrm{c}} \mathrm{dm}^{-3}\right)$ & 0,00 & $\operatorname{Ucc}\left(\mathrm{g} \mathrm{kg}^{-1}\right)$ & 78,1 \\
\hline $\mathrm{Ca}^{2+}\left(\mathrm{cmol}_{\mathrm{c}} \mathrm{dm}^{-3}\right)$ & 3,17 & $\operatorname{Upmp}\left(\mathrm{g} \mathrm{kg}^{-1}\right)$ & 42,7 \\
\hline $\mathrm{Mg}^{2+}\left(\mathrm{cmol}_{\mathrm{c}} \mathrm{dm}^{-3}\right)$ & 1,95 & Adi $\left(\mathrm{g} \mathrm{kg}^{-1}\right)$ & 70,5 \\
\hline $\mathrm{SB}\left(\mathrm{cmol}_{\mathrm{c}} \mathrm{dm}^{-3}\right)$ & 5,49 & Clas. textural & F. Arenoso \\
\hline $\mathrm{CTC}\left(\mathrm{cmol}_{\mathrm{C}} \mathrm{dm}^{-3}\right)$ & 8,00 & - & - \\
\hline $\mathrm{V}(\%)$ & 68,6 & - & - \\
\hline $\mathrm{MO}\left(\mathrm{g} \mathrm{kg}^{-1}\right)$ & 12,4 & - & - \\
\hline
\end{tabular}

*SB = soma de bases $\left(\mathrm{Ca}^{2+}+\mathrm{Mg}^{2+}+\mathrm{K}^{+}+\mathrm{Na}^{+}\right)$; $\mathrm{CTC}=$ capacidade de troca catiônica $\left[\mathrm{SB}+\left(\mathrm{H}^{+}+\mathrm{Al}^{3+}\right) ; \mathrm{V}=\right.$ saturação por bases trocáveis $(\mathrm{SB} / \mathrm{CTC}) 100 ; \mathrm{MOS}=$ matéria orgânica do solo; $\mathrm{DS}=$ densidade do solo; $\mathrm{DP}=$ densidade de partículas; Ucc = umidade do solo na capacidade de campo; Upmp = umidade do solo no ponto de murcha permanente; Adi $=$ Argila dispersa em água; F. Arenoso $=$ Franco Arenoso. Fonte: Autores.

Para a pesquisa foi escolhida uma área cultivada com Nopalea cochenillifera Salm-Dyck de dois anos de idade, com uma densidade de 20.000 plantas ha-1 $(1 \times 0,5 \mathrm{~m}$, entre fileiras e plantas, respectivamente). Antes da instalação do experimento realizou-se um corte nas plantas, preservando os cladódios primários para a manutenção do estande. Após o início do experimento foi realizada uma adubação com base nos resultados da análise de solo (Tabela 1) e da respectiva recomendação sugerida por Santos et al. (2008) para a cultura, periodicamente foram realizados tratos culturais, na forma de capina com enxada, em toda a área cultivada.

Os tratamentos foram distribuídos em blocos ao acaso, com três repetições e quinze plantas por parcela, com esquema fatorial $2 \times 4$, correspondente ao solo sem e com cobertura morta e quatro horários de avaliação da fluorescência da Clorofila 
a, 08:00, 11:00, 14:00 e 17:00 horas. A cobertura morta foi composta por ervas espontâneas retiradas da área experimental e adjacências após capina manual, com espessura de $5 \mathrm{~cm}$, distribuída em toda a parcela experimental.

As plantas foram avaliadas quanto a sua emissão de fluorescência da clorofila a, de acordo com a metodologia proposta por Maxwell e Johnson (2000). Realizou-se leituras em duas plantas de cada parcela e em dois cladódios maduros de cada planta, caracterizando-se por sustentar de um a dois cladódios jovens. As áreas dos cladódios foram adaptadas ao escuro por 30 minutos utilizando-se clipes foliares (Rohácek, 2002; Nunes et al. 2017). Foram analisadas as variáveis: fluorescência inicial (F0), fluorescência variável (FV), fluorescência máxima (FM), relação FV/F0 e rendimento quântico potencial (FV/FM). Para as medições, foi utilizado um fluorômetro portátil de excitação contínua (Sciences Inc.- modelo OS-30p, Hudson, USA)

Os dados foram submetidos à análise de variância pelo teste $\mathrm{F}$, que é conclusivo para um grau de liberdade, como é o caso do fator cobertura morta. As médias referentes aos horários de avaliação foram também submetidos ao teste $\mathrm{F}$ e quando apresentaram efeito significativo foram comparadas entre si pelo teste de Tukey a 5\% de probabilidade. Para as análises dos dados utilizou-se o software estatístico Sisvar (Ferreira, 2011).

\section{Resultados e Discussão}

Observa-se que independentemente do horário de avaliação, a fluorescência inicial (F0) das plantas de palma forrageira cultivadas no solo ausente de cobertura morta não apresentou diferenças significativas, entretanto, no solo coberto com resíduos vegetais, os maiores valores foram alcançados nos horários de 8:00 e 11:00 da manhã. Na comparação entre os tipos de manejo do solo, verificou-se que o uso da cobertura elevou em 30\% a F0 às 8:00 da manhã (Figura 1).

Figura 1. Fluorescência inicial de N. cochenillifera Salm-Dyck sob diferentes manejos de solo e horários de avaliação.

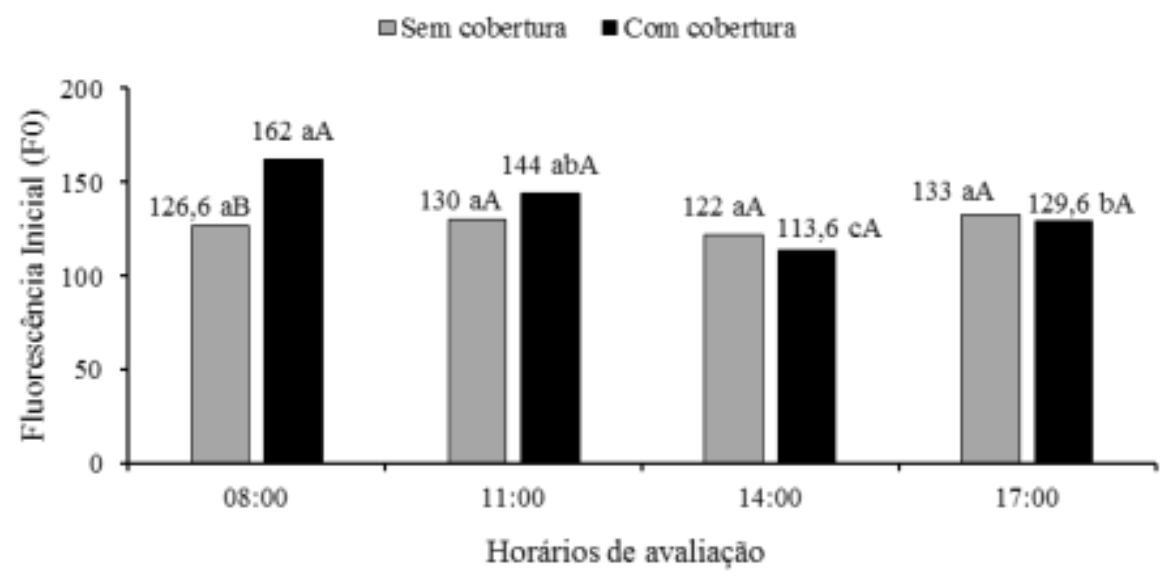

*Médias seguidas pela mesma letra não diferem entre si pelo teste de Tukey a 5\% de probabilidade. Letras minúsculas comparam os manejos de solo em todos os horários de avaliação; letras maiúsculas comparam os manejos de solo dentro de cada horário. Fonte: Autores.

A Fluorescência inicial é caracterizada como a mínima fluorescência emitida quando todos os centros de reação, adaptados ao escuro, estão abertos (Gorbe \& Calatayud, 2012). Maiores valores de F0 podem estar associados a perda dos centros de reação do Fotossistema II e danos no âmbito oxidativo, uma vez que eles já estariam indisponíveis para a recepção de energia quântica, causando diminuição na capacidade de transferir energia, devido ao desprendimento do complexo coletor de luz do complexo central desse fotossistema (Duarte, 2015; Lopes, 2016).

Com relação à fluorescência máxima $(\mathrm{Fm})$, verifica-se que, independentemente do tipo de manejo edáfico, sua maior intensidade foi alcançada às 17:00 horas, com superioridade de pelo menos 43,8\% e 34,1\% para as plantas do solo sob 
ausência e presença de cobertura com resíduos vegetais (Figura 2). O uso da cobertura morta influenciou a Fm da palma forrageira apenas às 8:00 e 11:00 da manhã, elevando-a em 15,5 e 19,9\%, respectivamente.

Estes resultados indicam que ao longo do dia, a distribuição de energia fotoquímica entre os complexos coletores de luz do PSII, os centros de reação e o aceptor final do PSII da palma forrageira apresentaram diferenças consideráveis, ao pas so de serem elevados em mais de $78 \%$ na comparação entre o primeiro e último horário de avaliação no solo sem cobertura, podendo causar deficiência na fotorredução da quinona A (QA) e no fluxo de elétrons entre os fotossistemas das plantas estudadas (Tatagiba \& Pezzopane, 2007).

Figura 2. Fluorescência máxima de N. cochenillifera Salm-Dyck sob diferentes manejos de solo e horários de avaliação.

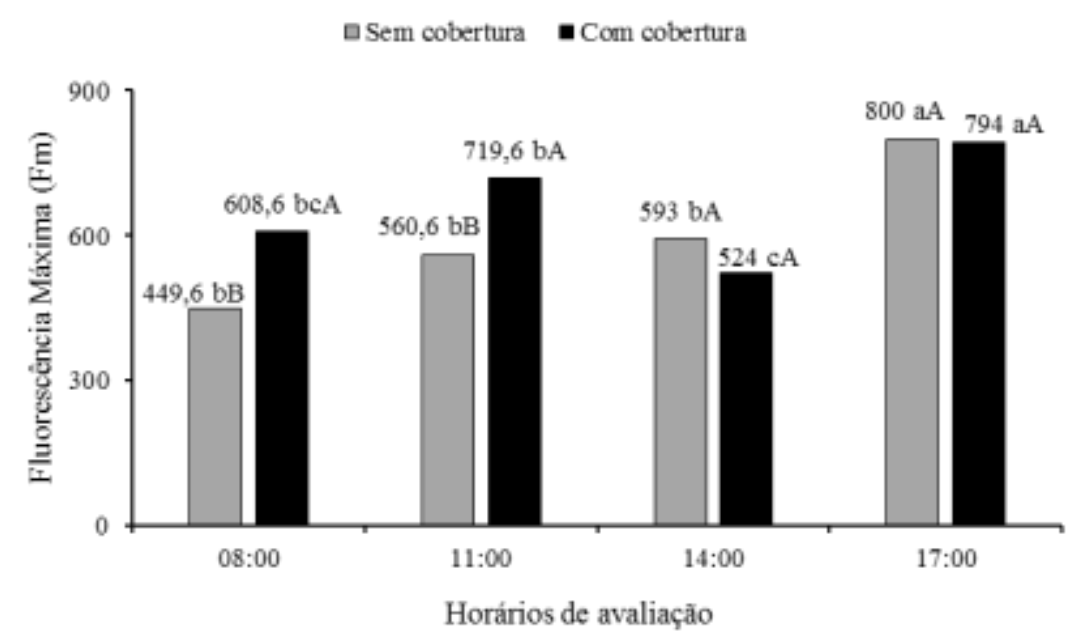

*Médias seguidas pela mesma letra não diferem entre si pelo teste de Tukey a 5\% de probabilidade. Letras minúsculas comparam os manejos de solo em todos os horários de avaliação; letras maiúsculas comparam os manejos de solo dentro de cada horário. Fonte: Autores.

A tendência observada para a Fm repetiu-se para a fluorescência variável (Fv), onde às 17:00 horas as plantas obtiveram os valores máximos, 667 e 664,3 elétrons quantum-1, respectivamente, quando cultivadas no solo sem e com cobertura morta, o que representa um aumento de até 106,5 e 48,7\% em comparação com os valores obtidos às 8:00 horas da manhã (Figura 3). A maior intensidade da Fm e Fv às 17:00 horas pode estar relacionada ao início do período da captação de $\mathrm{CO}_{2}$ por esta cactácea, que por possuir metabolismo ácido das crassuláceas (MAC), realiza este processo durante a noite, abrindo os estômatos ao final da tarde, indicando maior redução da quinona e fazendo com que os centros de reação atinjam sua capacidade máxima de reações fotoquímicas. 
Figura 3. Fluorescência variável de $N$. cochenillifera Salm-Dyck sob diferentes manejos de solo e horários de avaliação.

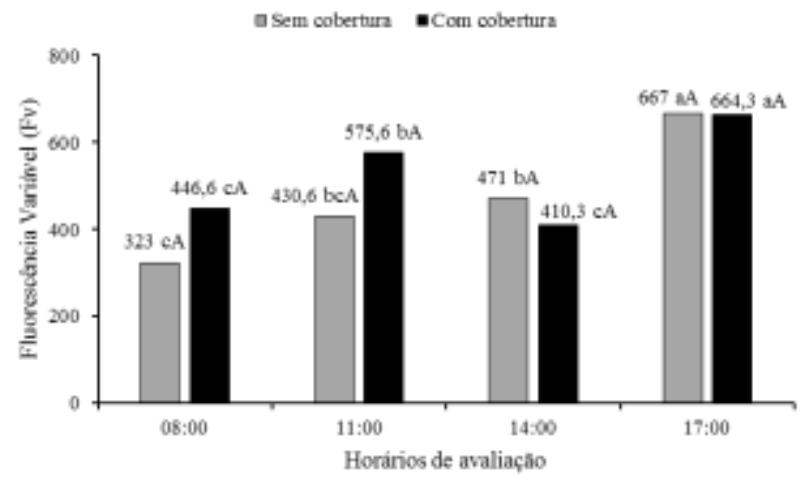

*Médias seguidas pela mesma letra não diferem entre si pelo teste de Tukey a 5\% de probabilidade. Letras minúsculas comparam os manejos de solo em todos os horários de avaliação; letras maiúsculas comparam os manejos de solo dentro de cada horário. Fonte: Autores.

Tendo-se estes dados como prerrogativa pode-se afirmar que as variáveis de fluorescência da clorofila a devem ser consideradas como indicativo da dinâmica comportamental desta cactácea em diferentes horários de avaliação. Neste sentido a Fv destaca-se por ser a diferença entre Fm e F0, representando uma expressiva adaptação do tecido fotossintentizante ao escuro, onde aumentos nos seus valores apontam elevação na capacidade do vegetal em transferir a energia dos elétrons ejetados das moléculas dos pigmentos fotossintéticos para formação do redutor NADPH e ATP (REIS \& CAMPOSTRINI, 2011; SOUZA et al., 2019).

A relação Fv/F0 foi mais elevada às 17:00 horas, 5,01 e 5,13 elétrons quantum-1, para o solo sob ausência e presença de cobertura morta, acréscimo de 97,2 e 87,2\% em comparação com as plantas analisadas às 08:00 horas. Esta relação não diferiu estatisticamente em função do uso de cobertura edáfica (Figura 4). Silva et al. (2015) ressaltam que esta razão pode ser entendida como um indicador de maior sensibilidade às variações nas taxas de conversão fotossintética, pois reflete instantaneamente à variações nos valores de F0 e/ou Fv.

Figura 4. Relação Fv/F0 de N. cochenillifera Salm-Dyck sob diferentes manejos de solo e horários de avaliação.

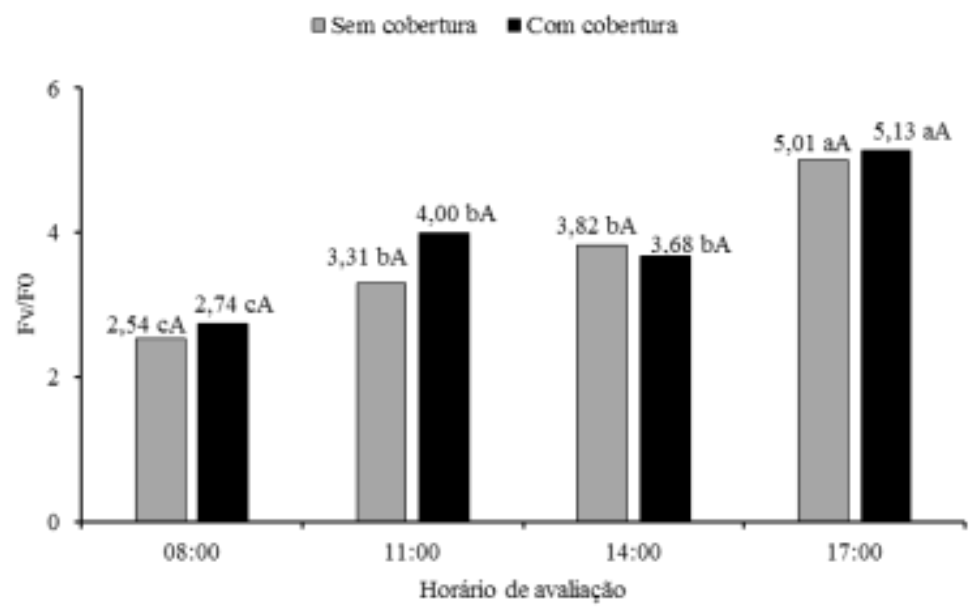

*Médias seguidas pela mesma letra não diferem entre si pelo teste de Tukey a 5\% de probabilidade. Letras minúsculas comparam os manejos de solo em todos os horários de avaliação; letras maiúsculas comparam os manejos de solo dentro de cada horário. Fonte: Autores. 
O rendimento quântico potencial nas plantas do solo ausente de cobertura foi mais elevado às 14:00 e 17:00 horas ( 0,79 e 0,83 elétrons quantum- 1 , respectivamente) entretanto no solo coberto com resíduos vegetais, os maiores valores médios foram verificados às 11:00 e às 17:00 (0,80 e 0,83 elétrons quantum-1). Não se verificou efeito significativo do fator cobertura de solo sobre esta variável.

Figura 5. Rendimento quântico $(\mathrm{Fv} / \mathrm{Fm})$ de $N$. cochenillifera Salm-Dyck sob diferentes manejos de solo e horários de avaliação.

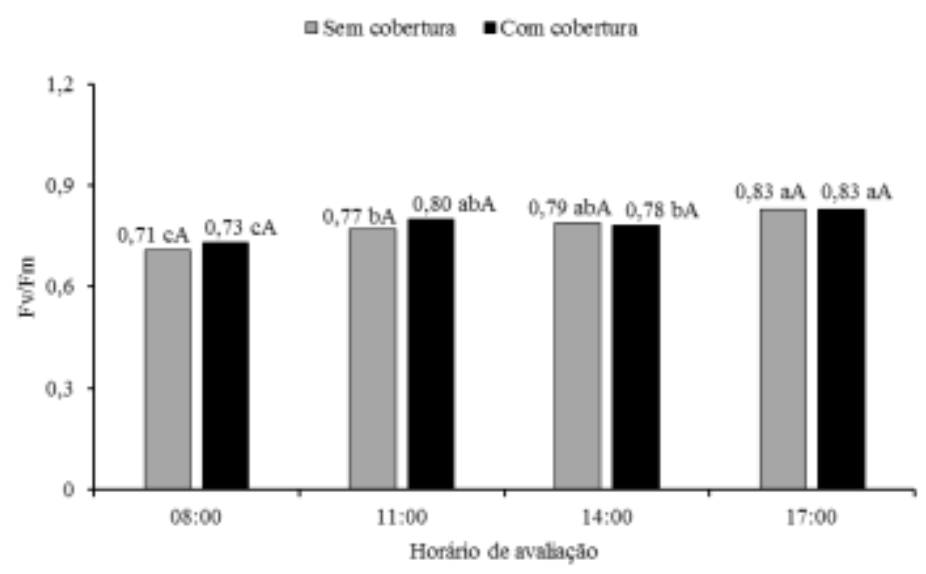

*Médias seguidas pela mesma letra não diferem entre si pelo teste de Tukey a 5\% de probabilidade. Letras minúsculas comparam os manejos de solo em todos os horários de avaliação; letras maiúsculas comparam os manejos de solo dentro de cada horário. Fonte: Autores.

Vieira et al. (2010) analisando fluorescência de abacaxizeiro, que assim como a palma forrageira é regido pelo metabolismo ácido das crassuláceas (MAC), averiguaram que os maiores valores de Fv/Fm foram às 6 e às 18 horas, e a menor foi às 10 horas, segundo estes autores, essas mudanças devem estar relacionadas às variações de temperatura ao longo do dia. Konrad et al. (2005) ressaltam que esta variável tem sido utilizada frequentemente para detectar perturbações no sistema fotossintético causadas por estresses ambientais e bióticos, visto que a diminuição indica inibição da atividade fotoquímica. Reis e Campostrini (2011) afirmam que plantas com Fv/Fm entre 0,75 e 0,85 elétrons quantum-1 estão com o aparelho fotossintético intacto e as que apresentam valores inferiores a 0,75 elétrons quantum-1 tem seu rendimento fotoquímico do fotossitema II comprometido, isto aponta que em ambos os tipos de manejo edáfico, as plantas analisadas às 8:00 horas foram ineficientes quando ao rendimento quântico.

\section{Conclusão}

O uso de cobertura morta elevou as fluorescências máxima e variável, especialmente nos primeiros horários de avaliação;

A atividade fotoquímica do fotossistema II foi mais elevada às 17:00 horas.

\section{Referências}

Almeida, I. V. B., Souza, J. T. A., \& Batista, M. C. (2019). Melhoramento genético de plantas forrageiras xerófilas: Revisão. PUBVET, 13 , 153.

Alvares, C. A., Stape, J. L., Sentelhas, P. C., Gonçalves, J. D. M., \& Sparovek, G. (2013). Köppen’s climate classification map for Brazil. Meteorologische Zeitschrift, 22(6), 711-728.

Borges, T. K. D. S., Montenegro, A. A. D. A., Santos, T. E. M. D., Silva, D. D. D., \& Silva Junior, V. D. P. (2014). Influência de práticas conservacionistas na umidade do solo e no cultivo do milho (Zea mays L.) em semiárido nordestino. 
Research, Society and Development, v. 10, n. 9, e40010918165, 2021

(CC BY 4.0) | ISSN 2525-3409 | DOI: http://dx.doi.org/10.33448/rsd-v10i9.18165

Duarte, H. H. F (2015). Status hídrico, fluorescência da clorofila e trocas gasosas em pimentão cultivado sob estresse salino. Dissertação (Mestrado em Ciências do Solo), Universidade Federal Rural de Pernambuco. Recife. 58 f.

Ferreira, D. F. (2011). Sisvar: a computer statistical analysis system. Ciência e agrotecnologia, 35, 1039-1042.

Gorbe, E., \& Calatayud, A. (2012). Applications of chlorophyll fluorescence imaging technique in horticultural research: a review. Scientia Horticulturae, $138,24-35$.

Konrad, M. L. F., Silva, J. A. B. D., Furlani, P. R., \& Machado, E. C. (2005). Trocas gasosas e fluorescência da clorofila em seis cultivares de cafeeiro sob estresse de alumínio. Bragantia, 64, 339-347.

Lopes, M. N. (2016). Ecofisiologia, nutrição e análise econômica da palma forrageira sob diferentes manejos no semiárido brasileiro.

Maxwell, K., \& Johnson, G. N. (2000). Chlorophyll fluorescence—a practical guide. Journal of experimental botany, 51(345), 659-668.

Nunes, J. C., Cavalcante, L. F., Pereira, W. E., Souza, J. T. A., de Almeida, D. J., Oresca, D., \& Fernandes, P. D. (2017). Gas exchange and productivity of yellow passion fruit irrigated with saline water and fertilized with potassium and biofertilizer. International Journal of Agriculture and Natural Resources, 44(2), 168-183.

Reis, F. O., \& Campostrini, E. (2011). Microaspersão de água sobre a copa: um estudo relacionado às trocas gasosas e à eficiência fotoquímica em plantas de mamoeiro. Revista brasileira de agrociencia, 17(3), 284-295.

Ribeiro, J. E. S., Figueiredo, F. R. A., dos Santos Coêlho, E., \& de Albuquerque, M. B. (2019). Morphophysiological aspects of young Calotropis procera plants submitted to different shading levels. Scientia Plena, 15(11).

Roháček, K. (2002). Chlorophyll fluorescence parameters: the definitions, photosynthetic meaning, and mutual relationships. Photosynthetica, 40(1), 13-29.

Santos, D. C., Lira, M. A., Dubeux Júnior, J. C. B., Santos, M. V. F., Mello, A. C. L. (2008) Recomendação de adubação para a palma forrageira. In: Cavalcanti, F. J. A. (Ed.) Recomendações de adubação para o estado de Pernambuco: $2^{a}$ Aproximação. Instituto Agronômico de Pernambuco, Recife-PE. p. 178 .

Santos, M. R., Silva, A. J. P., Fonceca, V. A., Campos, A. R. F., Lisboa, M. A. (2017) Irrigação na palma forrageira. Informe Agropecuário, 38(296): 76-90.

Solos, E. (2013). Sistema brasileiro de classificação de solos. Centro Nacional de Pesquisa de Solos: Rio de Janeiro.

Souza, J. A., da Silva Ribeiro, J. E., de Farias Ramos, J. P., de Sousa, W. H., Araújo, J. S., Lima, G. F. C., \& Dias, J. A. (2019). Rendimento quântico e eficiência de uso da água de genótipos de palma forrageira no Semiárido brasileiro. Archivos de zootecnia, 68(262), 268-273.

Sun, Q., Wang, Y., Chen, G., Yang, H., \& Du, T. (2018). Water use efficiency was improved at leaf and yield levels of tomato plants by continuous irrigation using semipermeable membrane. Agricultural Water Management, 203, 430-437.

Taiz, L., Zeiger, E., Møller, I. M., \& Murphy, A. (2017). Fisiologia e desenvolvimento vegetal. Artmed Editora.

Tatagiba, S. D., \& Pezzopane, J. E. M. (2007). Cinética de emissão de fluorescência das clorofilas em dois clones de Eucalyptus. Revista Científica Eletrônica de Engenharia Florestal, 10(12), 1-14.

Vieira, D. A. D. P., Portes, T. D. A., Stacciarini-Seraphin, E., \& Teixeira, J. B. (2010). Fluorescência e teores de clorofilas em abacaxizeiro cv. Pérola submetido a diferentes concentrações de sulfato de amônio. Revista Brasileira de Fruticultura, 32, 360-368. 